MIDPI

sciforum
MOL2NET, International Conference Series on Multidisciplinary Sciences

03. SRI-11: Summer Research Institute Symposium, MDC, Miami, FL, USA, 2019

\title{
Guarana and nanotechnology
}

\author{
Leidi del Rosario Hernández Suárez
}

lhsuarez96@gmail.com

Department of Pharmacology, Faculty of Medicine, University of the Basque Country UPV/EHU, 48940, Leioa, Basque Country, Spain

Abstract. (mandatory)

Guarana or Paullinia cupana var. Sorbilis (Mart.) Ducke, since it has relevant therapeutic effects, has been the subject of nanotechnology study in order to magnify its activity. In the article Protective effect of liposomes loaded with guarana on the hemolytic activity of author I Roggia, a study was conducted where physicochemical parameters were evaluated to determine the stability of liposomes containing guarana. To evaluate the hemolytic and antioxidant activity, the red blood cells of the rats were subjected to different formulations dissolved in PBS (Guarana liposomal, free form and liposomal guarana without guarana). These formulations were compared and the most stable results were those of one containing 1mgml-1 of guarana by the reverse phase evaporation method. On the other hand, the formulation of guarana in free form has dose-dependent antioxidant properties and the liposomal formulation without guarana hemolytic activity before polysorbate 80 present in the PBS of the buffer solution.

Keywords : Nanoparticle; Paullinia cupana

Bibliographic references: Coloides Surf B Biointerfaces. 11 de noviembre de 2019: 110636. doi: 10.1016 / j.colsurfb.2019.110636. 